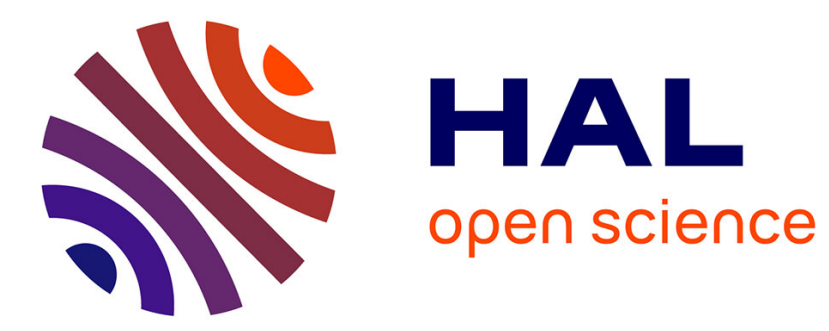

\title{
Outline of metabolic diseases in adult neurology
}

Fanny Mochel

\section{To cite this version:}

Fanny Mochel. Outline of metabolic diseases in adult neurology. Revue Neurologique, 2015, 171 (6-7), pp.531-538. 10.1016/j.neurol.2015.02.018 . hal-01161960

\section{HAL Id: hal-01161960 \\ https://hal.sorbonne-universite.fr/hal-01161960}

Submitted on 9 Jun 2015

HAL is a multi-disciplinary open access archive for the deposit and dissemination of scientific research documents, whether they are published or not. The documents may come from teaching and research institutions in France or abroad, or from public or private research centers.
L'archive ouverte pluridisciplinaire HAL, est destinée au dépôt et à la diffusion de documents scientifiques de niveau recherche, publiés ou non, émanant des établissements d'enseignement et de recherche français ou étrangers, des laboratoires publics ou privés. 
Outline of metabolic diseases in adult neurology

Aperçu des maladies neurométaboliques chez l'adulte

Fanny MOCHEL*, MD, PhD $(1,2,3,4)$

(1) AP-HP, Pitié-Salpêtrière University hospital, Department of Genetics, F-75013 Paris, France

(2) University Pierre and Marie Curie, F-75013 Paris, France

(3) AP-HP, Pitié-Salpêtrière University hospital, Neurometabolic Unit, F-75013 Paris, France

(4) Inserm U 1127, CNRS UMR 7225, Sorbonne Universités, UPMC Univ Paris 06 UMR S 1127, Institut du Cerveau et de la Moelle épinière, ICM, F-75013, Paris, France

\section{Corresponding author:}

Dr Fanny Mochel

Institut du Cerveau et de la Moelle épinière, Aile 4A

Groupe Hospitalier Pitié-Salpêtrière, 75013 Paris, France

Tel: +33 (0) 157274682 ;

Fax: +33 (0) 157274795

email: fanny.mochel@upmc.fr 


\section{Outline of metabolic diseases in adult neurology}

Aperçu des maladies neurométaboliques chez l'adulte

\section{Abstract}

Introduction: Inborn errors of metabolism (IEM) are traditionally defined by enzymatic deficiencies or defects in proteins involved in cellular metabolism. Historically discovered and characterized in children, a growing number of IEM are described in adults, and especially in the field of neurology. In daily practice, it is important to recognize emergency situations as well as neurodegenerative diseases for which a metabolic disease is likely, especially when therapeutic interventions are available.

Objectives: Here, the goal is to provide simple clinical, imaging and biochemical tools that can first orientate towards and then confirm the diagnosis of IEM. General guidelines are presented to treat the most common IEM during metabolic crises - acute encephalopathies with increased plasma ammonia, lactate or homocystein, as well as rhabdomyolysis. Examples of therapeutic strategies currently applied to chronic neurometabolic diseases are also provided - GLUT1 deficiency, adrenoleukodystrophy, cerebrotendinous xanthomatosis, Niemann-Pick type $C$ and Wilson disease. Genetic counseling is mandatory in some $X$-linked diseases - ornithine transcarbamylase deficiency and adrenoleukodystrophy - and recommended in maternally inherited mitochondrial diseases - mutations of mitochondrial DNA.

Conclusion: Besides these practical considerations, the contribution of metabolism to the field of adult neurology and neurosciences is much greater : first, with the identification of blood biomarkers that are progressively changing our diagnostic strategies thanks to lipidomic approaches, as illustrated in the field of spastic paraplegia and atypical psychiatric presentations; and second, through the understanding of physiopathological mechanisms involved in common neurological diseases thanks to the study of these rare diseases.

Key words: inborn errors of metabolism, biomarkers, therapeutics 


\section{Résumé}

Introduction : Les erreurs innées du métabolisme (EIM) correspondent classiquement à des déficits enzymatiques ou des altérations protéiques ayant un rôle dans le métabolisme cellulaire. Historiquement découvertes et décrites chez les enfants, un nombre croissant $d^{\prime} E I M$ sont identifiées chez les adultes, en particulier en neurologie. En pratique clinique, il est important de reconnaître les situations d'urgence et les maladies neurodégénératives pour lesquelles une maladie métabolique est probable, en particulier s'il existe des options thérapeutiques.

Objectifs : Le but ici est de décrire des outils cliniques, d'imagerie et biochimiques qui peuvent orienter puis confirmer le diagnostic d'EIM. Des recommandations générales sont proposées pour le traitement des décompensations des EIM les plus fréquentes encéphalopathies aigues avec hyperammoniémie, hyperlactacidémie ou hyperhomocystéinémie et rhabdomyolyse. Sont également présentés des exemples de stratégies thérapeutiques appliquées à des maladies neurométaboliques chroniques - déficit en GLUT1, adrénoleucodystrophie, xanthomatose cérébrotendineuse, Niemann-Pick de type C, maladie de Wilson. Le conseil génétique est nécessaire dans certaines pathologies liées à I'X - déficit en ornithine transcarbamylase et adrénoleucodystrophie - et recommandé dans les maladies mitochondriales avec hérédité maternelle - mutations de l'ADN mitochondrial. Conclusion : En dehors de ces considérations pratiques, la contribution du métabolisme au domaine de la neurologie et des neurosciences est bien plus grande : d'abord avec l'identification de biomarqueurs sanguins qui changent progressivement nos stratégies diagnostiques grâce à l'essor de la lipidomique, notamment dans le domaine des paraparésies spastiques et des présentations psychiatriques atypiques; mas aussi grâce à la compréhension de mécanismes physiopathologiques dans des maladies neurologiques communes au travers de l'étude de ces maladies rares.

Mots clés: erreurs innées du metabolism, biomarqueurs, traitement. 


\section{What is a neurometabolic disease?}

Among the growing list of genetic diseases, inborn errors of metabolism (IEM) are historically defined by enzymatic deficiencies or defects in proteins involved in cellular metabolism. This implies the use of specific strategies to diagnose and treat IEM. Likewise, before the confirmation by molecular analyses, the diagnostic of several IEM can be greatly facilitated by biochemical testing showing abnormal metabolites concentration or enzymatic activities. The recognition of IEM is even more important as some of them can be treated through enzyme replacement therapy or cofactors that increase enzymatic activity, the provision of deficient metabolites, the inhibition of accumulating compounds and/or specific dietary interventions. IEM have been traditionally classified as (i) disorders of intermediary metabolism comprising both intoxication defects, related to the accumulation of a toxic metabolic like hyperammonemia in urea cycle defects, or energetic defects such as respiratory chain deficiencies; (ii) disorders of organelles like lysosomal or peroxisomal diseases as well as congenital disorder of glycosylation; and (iii) disorders affecting neurotransmitters and metals such as monoamines synthesis, neurodegeneration with brain iron accumulation (NBIA) and Wilson disease.

However, this traditional approach of IEM is currently challenged by the discovery of novel neurogenetic entities thanks to next generation sequencing techniques and a better understanding of physiopathological mechanisms underlying IEM. For example, the so-called intoxication defects are in fact commonly associated with energy deficiency - e.g. deficiency of brain creatine in urea cycle defects. Conversely, toxic metabolites like nitric oxide are likely important actors in energetic defects such as mitochondrial diseases and especially MELAS syndrome (myopathy, encephalopathy, lactic acidosis and stroke-like episodes). The current classification of IEM based on cellular organelles - i.e. mitochondrial, lysosomal, peroxisomal disorders - is also challenged by the delineation of a new group of disorders affecting the synthesis and remodeling of complex lipids that involve several cellular compartments [1]. Furthermore, it becomes difficult to distinguish neurometabolic diseases among other neurogenetic entities. Mutations in genes involved in dominant forms of optic atrophy (OPA1) or recessive forms of spastic paraplegia (SPG7) have now been identified in mitochondrial diseases that associate peripheral neuropathy, cerebellar ataxia, external ophthalmoplegia with multiple mitochondrial DNA deletion on muscle biopsy [2]. The 
possibility for a given gene to be associated with both dominant (heterozygous mutation) and recessive (homozygous or composite heterozygous mutations) inheritance is, as a matter of fact, reported in a growing number of neurometabolic disorders [3]. Therefore, while certain biochemical tools are preferentially used for the diagnosis of certain subgroups of IEM - i.e. ammonemia in urea cycle defects -, it seems increasingly relevant to identify common cellular pathways between metabolic partners - such as OPA1 and SPG7 which proteins are both localized at the internal mitochondrial membrane - in order to gain knowledge in the pathophysiology underlying adult neurometabolic diseases. Several reviews on IEM in adults have been published $[4,5]$. The purpose of this outline is to provide simple diagnostic tools and therapeutic strategies to address the most frequent IEM in adult neurology.

\section{What are the tools useful for the diagnosis of neurometabolic diseases?}

First, one should be pay much attention to the clinical history and signs of the disease. This starts with the family history. For example, unexplained comas in men should prompt to search for an ornithine transcarbamylase deficiency, a X-linked disorder and the most common urea cycle defect. A history of disabling migraine and/or type 1 diabetes in mothers of patients with complex neurological phenotypes is evocative of mitochondrial DNA mutations, which are maternally inherited mutations and a common cause of mitochondrial diseases in adults. As for patient medical history, an early disease onset (childhood, adolescence) and/or the recurrence of symptoms during stress (infection, generalized anesthesia, trauma, prolonged fasting) need attention and should motivate investigations of intermediary metabolism. The worsening of movement disorders during exercise suggests GLUT1 deficiency, a glucose transporter defect into the brain. Some clinical associations are also very informative, especially neurological symptoms associated with (i) digestive symptoms (abdominal pain, nausea, vomiting) in intoxication defects like urea cycle diseases and acute porphyria; (ii) psychotic symptoms and/or cataract in cerebrotendinous xanthomatosis; (iii) ichthyosis in peroxisomal diseases and Sjögren-Larsson disease; (iv) splenomegaly and/or supranuclear ophthalmoplegia in Gaucher disease and Niemann-Pick type C; (v) thrombosis and/or lens dislocation in homocystinuria; (vi) subacute combined degeneration of the spinal cord in homocysteine remethylation defects. 
Next to clinical tools, MRI of the brain and spine can be key to the diagnosis of neurometabolic diseases in adults. During acute encephalopathies, necrotic lesions of the basal ganglia - T2 hyperintensity of the putamen, caudate - and/or thalami and the brainstem, called Leigh syndrome (Figures 1a and 1b), or cortico-subcortical lesions with increased apparent diffusion coefficient, called pseudo-strokes (Figure 1c and 1d), are highly suggestive of a mitochondrial energy deficit. Among chronic neurodegenerative diseases, especially when associated with movement disorders and/or psychiatric symptoms, diffuse T2 and FLAIR hyperintensities of the basal ganglia, the thalami, the mesencephalon and the dentate nucleus should prompt to search for Wilson disease (ATP7B mutations) by measuring plasma and urine copper as well as plasma ceruloplasmin levels. Furthermore, T1 hyperintensities of the basal ganglia, especially the globus pallidus, and sometimes the midbrain and cerebellum, in the context of dystonia and/or parkinsonism are highly evocative of SLC3OA10 mutations. This newly recognized neurometabolic entity is associated with hypermanganesemia, polycythemia, chronic liver disease and can be efficiently treated with chelation therapy and iron supplementation. Conversely, T2 and FLAIR hypointensity of the globus pallidus is a strong argument for NBIA due to iron overload [6]. The most common forms of NBIA are mutations of PLA2G6 and c19orf12 characterized by a T2 hypointensity of the globus pallidus and the substantia nigra (Figures $2 \mathrm{a}$ and $2 \mathrm{~b}$ ). PANK2 mutations are often associated with a T2 central hyperintensity surrounded by hypointensity centered on the globus pallidus, the so-called "eye of the tiger". WDR45 mutations are characterized by a T1 hyperintense halo that surrounds the substantia nigra in addition to the T2 hypointensity of the globus pallidus and the substantia nigra (Figures $2 \mathrm{c}$ and $2 \mathrm{~d}$ ). Although a common feature of NBIA is represented by iron accumulation, the biochemical pathways underlying these genetic defects rather involve lipid metabolism and membrane/organelles (mitochondria) remodeling, corroborating the idea that iron deposition may be only a secondary phenomenon [7]. Brain MRI is also critical to identify metabolic white matter diseases [8]. Unlike leukoencephalopathies of vascular or inflammatory origin, adult leukodystrophies often present with a mild, bilateral, symmetric and, often, fascicular hyperintensity of the white matter on T2 and FLAIR sequences. Among the most common late onset forms of leukodystrophy, hyperintensities restricted to the corticospinal tracts are evocative of Krabbe disease (i.e. galactocerebrosidase deficiency) and X-linked adrenoleukodystrophy (i.e. increased plasma very long chain fatty acids); 
hyperintensities of the periventricular white matter of a metachromatic leukodystrophy (i.e. arylsufatase 1 deficiency); hyperintensities of the dentate nucleus of a cerebrotendinous xanthomatosis (i.e. increased plasma cholestanol); a predominant cortical involvement that comprises the $U$ fibers of $L$ 2-hydroxyglutaric aciduria; a cystic breakdown of the white matter or cavitation on FLAIR sequences of $\mathrm{CACH}$ (childhood ataxia with central nervous hypomyelination); and hyperintensities of the internal and external capsules, the pyramidal tracts and medial lemniscus of the pons and medulla together with spine atrophy of adult polyglucosan body disease (i.e. glycogen branching enzyme deficiency). Spine atrophy is also a hallmark of leukodystrophies related to mutations of GFAP (Alexander disease), LMNB1 (duplication of lamin B1) and DARS2 (LBSL, leukoencephalopathy with brainstem and spinal cord involvement and lactate elevation). In vivo brain MRI spectroscopy may also provide some diagnostic insight such as in LBSL or some mitochondrial diseases with an increased lactate peak, cerebrotendinous xanthomatosis or Niemann-Pick type C with an increased choline peak, and Sjögren-Larsson disease with the abnormal presence of a lipid peak.

Besides MRI, the diagnosis of a neurometabolic disease can also be comforted by findings from electromyogram, fundoscopy and/or audiometry due to the frequent involvement of multiple systems in IEM. Overall, axonal neuropathies are more common in metabolic diseases resulting in energy deficiency - respiratory chain defects, pyruvate dehydrogenase deficiency, fatty acids oxidation defects, adult polyglucosan body disease - whereas demyelinating neuropathies are more suggestive of complex lipids metabolism metachromatic leukodystrophy, Krabbe disease, cerebrotendinous xanthomatosis [9]. Peroxisomal diseases also frequently manifest in adults with an axonal or demyelinating neuropathy associated with other neuropsychiatric symptoms. Of note, pure sensory neuronopathy are evocative of diseases affecting the maintenance of the mitochondrial DNA, and especially POLG mutations. In addition, ophthalmological abnormalities are commonly observed in mitochondrial diseases (optic atrophy, retinitis pigmentosa) and peroxisomal diseases (cataract, retinitis pigmentosa). An early onset cataract should also lead to the suspicion of cerebrotendinous xanthomatosis and a retinitis pigmentosa to a defect of homocystein remethylation pathways. Mitochondrial and peroxisomal diseases are often responsible for sensorineural deafness. 
As outlined above, unlike most genetic diseases, the diagnosis of neurometabolic diseases can be guided by dedicated biochemical analyses prior to molecular testing. In emergency, an unexplained encephalopathy must be explored using simple analyses such as plasma ammonia, lactate and homocystein. In case of confirmed hyperammonemia and/or hyperlactacidemia, specialized biochemical analyses should be performed in emergency: amino acids chromatography on plasma and urine plus urine orotic acid for urea cycle defects; plasma acylcarnitines profile and urine organic acids chromatography for organic aciduria and fatty acids oxidation defects. In case of confirmed hyperhomocystinemia, the metabolic workup should include plasma vitamin B12 and folate to rule out a deficiency in these vitamins. Plasma methionine and urine methylmalonic acid (MMA) are then needed to discriminate between cobalamin $\mathrm{C}$ deficiency (low methionine, high MMA), cystathionine beta-synthase deficiency (high methionine, normal MMA) and a deficiency of 5,10methylenetetrahydrofolate reductase (low methionine, normal MMA). If an energy deficiency is suspected, CSF analyses can be useful with the measurement of lactate (respiratory chain defects), pyruvate (pyruvate dehydrogenase deficiency) and glucose (GLUT1 deficiency, low CSF glucose $<2.2 \mathrm{mmol} / \mathrm{I}$ and low CSF/plasma glucose ratio $<0.45$ ). When suspecting a mitochondrial oxidative phosphorylation defect, several lines of evidence can be gathered: (i) increased plasma and/or CSF lactate levels together with an increased lactate/pyruvate ratio (>20); (ii) immunohistochemical analyses on muscle with decreased cytochrome oxidase activity and/or increased succinate dehydrogenase activity and/or ragged red fibers: (iii) an isolated or combined deficiency of the respiratory chain complexes on muscle, more often than cultured fibroblasts in adults; and (iv) single or multiple mitochondrial DNA deletions on muscle, the latter finding suggesting mutations of genes involved in mitochondrial DNA maintenance such as POLG. However, normal lactate and normal immunohistochemical and biochemical analyses cannot rule out a mitochondrial disease in adults. Conversely, mild to moderate muscle abnormalities can be seen in adults over 50 years old without any primary mitochondrial dysfunction. Overall, mitochondrial DNA deletions and mutations as well as POLG mutations are the most commonly diagnosed mitochondrial diseases in adults. Furthermore, when suspecting a peroxisomal disease, the minimal biochemical assessment should include plasma very long chain fatty acids, phytanic and pristanic acids. Late onset peroxisomal defects may be associated with a normal biochemical profile. If the clinical suspicion of a peroxisomal defect is strong, then a catalase 
staining test on fibroblasts should be performed. Besides these biochemical analyses dedicated to the exploration of given organelles, other important plasma biomarkers are available in neurology practice. The exploration of spastic paraplegia should comprise the measurement of plasma very long chain fatty acids for the diagnosis of an adrenomyeloneuropathy, a $\mathrm{X}$ linked disorder due to $A B C D 1$ mutation, as well as plasma 27and 25-hydroxycholesterol levels for the diagnosis of spastic paraplegia type 5 , a recessive disorder due to CYP7B1 mutations. Atypical psychotic symptoms, especially when associated with hallucinations and/or poorly responsive to treatments, should motivate the measurement in plasma of cholestanol for the diagnosis of cerebrotendinous xanthomatosis, a recessive disorder due to CYP27A1 mutations, and 7-ketocholesterol (now replacing the filipin staining test on fibroblasts) for the diagnosis of Niemann-Pick type $C$, a recessive disease due to NPC1 (95\%) and NPC2 mutations [10].

Last, but not least, there are few IEM that can be diagnosed thanks to simple therapeutic tests. This is the case of patients harboring mutations in the SLC19A3 gene, which encodes the transmembrane thiamine transporter hTHTR2. Mutations in this gene cause the socalled biotin-responsive basal ganglia disease (BBGD) and lead to recurrent episodes of encephalopathy that can be life-threatening or result in severe dystonia if untreated. Brain $\mathrm{MRI}$ is remarkable due to the presence of lesions of the caudate nuclei and putamen, as well as the mesencephalon and cortical-subcortical areas. Treatment with high doses of biotin and thiamine can result in spectacular clinical and radiologic improvement, even in adults [11]. Autosomal dominant dopa-responsive dystonia (DRD), mainly due to mutations in the GCH1 gene that encodes GTC cyclohydrolase I, a rate-limiting step in tetrahydrobiopterin biosynthesis, is another good example. Likewise, patients who present with generalized dystonia, sometimes severely disabling, experience a dramatic and sustained response (complete or near-complete responsiveness of symptoms) to relatively low doses of orally administered levodopa [12].

\section{What are the neurometabolic diseases requiring an emergency treatment?}

In emergency, there are 2 main situations when an IEM should be strongly considered: unexplained acute encephalopathy and rhabdomyolysis. As detailed above, the clinical history of the patient and his/her family, simple biochemical analyses to be repeated in 
emergency (ammonemia, lactacidemia, homocysteinemia) and, sometimes, brain MRI are instrumental to guide the diagnostic and therefore therapeutic strategies. As mentioned above, recurrent episodes of encephalopathy with T2 hyperintensities of the caudate and putamen should prompt the administration of high doses of biotin and thiamine due to the suspicion of BBGD. In case of an encephalopathy with hyperammoniemia, a personal or familial anamnesis of recurrent confusional state and/or a metabolic trigger, the cooccurrence of digestive and neuropsychiatric symptoms, the dissociation between liver insufficiency and relatively mild cytolysis are very evocative of an urea cycle defect, and especially ornithine transcarbamylase deficiency. Plasma amino acids chromatography and urine orotic acid should be performed in emergency to identify the defect in urea cycle enzymes but should not delay the initiation of treatment. Treatment must include: a temporary $(<72 \mathrm{~h}$ in adults) protein removal from intake (oral, enteral or parenteral nutrition), a reversal of the catabolic state through caloric supplementation using IV glucose and lipids (2500 to 3000 calories minimum for women and men respectively) which often requires a central IV line, pharmacologic scavenging of excess nitrogen using sodium benzoate and/or sodium phenylbutyrate and IV arginine to replace arginine not produced by the urea cycle and prevents its deficiency from causing additional protein catabolism. Depending on ammonia levels at baseline, the severity of the neurological status and/or the kinetics of ammonia upon medical treatment, dialysis may be necessary for a few hours to remove ammonia. For ornithine transcarbamylase deficiency, specialized genetic counseling is mandatory as the disease can be transmitted from generation to generation. As a X-linked disease, men are more severely affected than women but women can become symptomatic during pregnancy or in the post-partum period. In addition, a late onset form does not preclude more severe and early onset forms in other family members carriers of the mutation. Likewise, genetic counseling should lead to the identification of all possible family carriers, assess their protein tolerance and provide emergency recommendations during metabolic crises in order to avoid life-threatening episodes. In case of an encephalopathy with hyperlactacidemia, pyruvate levels are important to determine the lactate/pyruvate ratio : in pyruvate dehydrogenase deficiency, the ratio is normal due to concomitant elevation of pyruvicemia - a ketogenic diet as well as thiamine supplementation may be initiated in emergency, whereas in oxidative phosphorylation defects, the lactate/pyruvate ratio is elevated. Clinical features (ptosis, ophthalmoplegia, sensory neuronopathy) and/or 
MRI findings (Leigh syndrome, pseudo-strokes) are also important to orientate towards a mitochondrial energy deficiency. Except for status epilepticus or intractable seizures for which a ketogenic diet is indicated, there are unfortunately no drug that have proved efficacy in mitochondrial diseases. However, the initiation of some treatments must be avoided, like valproate, as they can aggravate the metabolic status. The implication of other systems (kidneys, liver) must also be considered, especially cardiac dysfunction as they can significantly worsen disease prognosis. For mutations of mitochondrial DNA, genetic counseling is also important as the mitochondrial DNA is exclusively transmitted by mothers. For the most common mitochondrial DNA mutations (MELAS, MERRF mutations), the risk to develop symptoms usually depends on the proportion of mutations in the target tissues, a phenomenon called heteroplasmy. Genetic counseling and prospective follow-up of individuals at risk should be performed by expert genetic centers. In case of an encephalopathy with hyperhomocystinemia, an anamnesis of thrombotic events, subacute combined degeneration of the spinal cord and/or psychiatric symptoms are further arguments to suspect a homocystein remethylation defect. Regardless of the diagnosis (cobalamin C, cystathionine beta-synthase or 5,10-methylenetetrahydrofolate reductase deficiency, see above), an emergency treatment using a combination of methylating compounds such as betaine, folate, B12 and B6 vitamins may lead to a prompt neurological improvement. Of note, even if they usually present as episodes of acute abdominal pain with sympathetic signs rather than confusional state, psychotic symptoms or seizures, acute porphyria are easily diagnosed with increased urinary porphyrins and treated using glucose infusion as well as perfusion of heme arginate.

In case of rhabdomyolysis, besides McArdle disease (muscle glycogen phosphorylase deficiency) characterized by exercise intolerance and the "second wind" phenomenon, fatty acids oxidation defects should be ruled out because of a high mortality rate due to heart conduction defects and profound hypoglycemia. Plasma acylcarnitines profile and urine organic acids chromatography will lead to the diagnosis but should not delay an emergency treatment that primarily consists in perfusing high doses of glucose in order to stop lipolysis and provide urgent energy substrates for the liver as well as dialysis when rhabdomyolysis leads to an acute renal failure. 


\section{What are the other treatable neurometabolic diseases?}

Besides these emergency situations, other neurometabolic diseases are amenable to treatments and can require genetic counseling. As stated above, any patient presenting with generalized dystonia should benefit from a therapeutic test with levodopa in the event of DRD. Moreover, in GLUT1 deficiency, SLC2A1 mutations usually occurs de novo but a dominant family history should be carefully looked for and genetic testing offered to persons at risk as treatment is available. Due to the insufficient provision of glucose to astrocytes, the disease can manifest in children with a predominant epileptic encephalopathy whereas recurrent episodes of movement disorders, especially during exercise or fast, are common when patients become adults or in late onset forms of the disease [13]. The alternative provision of energy thanks to high levels of ketone bodies, using a ketogenic diet, leads to a very good response on seizures and a partial response on movement disorders. Other therapeutic options are under development to provide alternative brain energy substrates in GLUT1 deficiency. $A B C D 1$ mutations are in majority responsible for adrenomyeloneuropathy, i.e. spastic paraplegia and/or peripheral neuropathy, in both men and women whereas the cerebral form of the disease, called adrenoleukodystrophy, is characterized by a severe and rapid motor and cognitive decline, which exclusively occurs in men - usually during infancy but cases have been reported up to 50 years old. Adrenal insufficiency is also predominantly seen in men. The cerebral and adrenal forms of this X-linked disease motivate a thorough genetic counseling in order to identify men under 50 years of age at risk of being carriers. The identification of inflammatory lesions on brain MRI before symptoms onset is a good indication for bone marrow transplant that can prevent the evolution of the disease towards a devastating cerebral form. Of note, X-linked adrenoleukodystrophy is also the first neurometabolic disease for which gene therapy has recently been successful [14]. Cerebrotendinous xanthomatosis and Niemann-Pick type $C$ are 2 other metabolic diseases involved in complex lipids metabolism with therapeutic perspectives. In both, the association of psychiatric symptoms with spastic ataxia or cerebellar ataxia should motivate the measurements of plasma cholestanol and 7-ketocholesterol respectively. In cerebrotendinous xanthomatosis, despite the frequent occurrence of diarrhea since infancy as well as cataract during childhood or adolescence, the diagnosis is almost always done in adults. Treatment with chenodeoxycholic acid leads to the normalization of cholestanol levels within a few months as well as a rapid improvement of digestive and psychiatric 
symptoms. Chenodeoxycholic acid has also been reported to stabilize peripheral neuropathy on the long term and improve motor functions [15]. In Niemann-Pick type C, substrate reduction therapies, like miglustat an inhibitor of glucosylceramide synthase, reduce the accumulation of glycosphingolipids and seem to stabilize some components of the disease like oculomotor functions and swallowing abilities [16]. Other therapeutic options are under development to counteract glycosphingolipids accumulation in Niemann-Pick type C. Reducing agents like copper or manganese chelating drugs are also the therapeutic strategies of choice in Wilson disease or SLC3OA10 mutations respectively. Likewise, penicillamine or trientine increase urinary excretion of copper and can reduce hepatic, neurologic, and psychiatric findings in many symptomatic individuals as well as prevent symptoms onset in presymptomatic individuals.

\section{Conclusion}

Initially discovered and described in children, a growing number of IEM are now diagnosed in adults, especially in the field of neurology. Besides the neurometabolic diseases that usually manifest only in adults (i.e. adult polyglucosan body disease), IEM that predominantly manifest in childhood tend to have a different clinical and imaging phenotype in adults, such as CYP7B1 mutations presenting as a severe liver disease in infants but a progressive spastic paraplegia in adults [17], explaining why adult physicians dedicated to the diagnosis and care of these patients are specifically needed. Far from being exhaustive, this outline aims at providing simple diagnostic tools and major therapeutic strategies needed to recognize and treat the most common adult neurometabolic diseases that are likely to be seen in neurology practice. The contribution of metabolism to the field of adult neurology and neurosciences is however much greater. Besides the existing blood biomarkers cited above that are progressively changing our approach to clinical diagnoses of spastic paraplegia or atypical psychiatric symptoms for example, it is likely that additional biomarkers will be identified in the soon future thanks to the development of lipidomic approaches. This may be especially true for the field of inherited spastic paraplegia as several recently identified genes are involved in the remodeling of phospholipids - SPG54 (DDHD1), SPG28 (DDHD2), SPG39 (NTE), SPG56 (CYP2U1) - and the biosynthesis of sphingolipids - SPG35 (FA2H), SPG46 (GBA2), SPG26 (B4GALNT1) - opening the way to a novel class of IEM $[1,18]$. These rare diseases also shed light on mechanisms involved in 
common neurological diseases or symptoms such as, for spasticity, the importance of the maintenance of lipid membrane homeostasis. Another important example is the identification of heterozygous mutations in the GBA1 gene, encoding lysosomal glucocerebrosidase, as the first risk factor of sporadic Parkinson disease [19]. More than a genetic factor, it appears that the decrease of glucocerebrosidase activity itself can favor the development of a synuclein-related pathology like seen in Parkinson disease. Indeed, the accumulation of lysosomal glucosylceramides due to glucocerebrosidase deficiency seems to accelerate and stabilize soluble alpha-synuclein oligomers, which eventually convert into amyloid fibrils [20]. This has important consequences on the development of therapeutic strategies in Parkinson disease as increasing the activity of glucocerebrosidase could be neuroprotective. Single observations in IEM can also contribute to a better understanding of brain physiology. Likewise, in adult polyglucosan body disease, we showed that abnormal glycogen in astrocytes is sufficient to cause motor dysfunction and a severe leukodystrophy [21]. This comes to support an important role of not only glucose and ketone bodies in brain energy metabolism but also glycogen as suggested by other neurogenetic disorders affecting glycogen metabolism such as Lafora disease. 


\section{Acknowledgements}

The author wishes to thanks colleagues from the Neurometabolic Unit at the PitiéSalpêtrière University hospital, especially Dr Foudil Lamari, Dr Claude Jardel, Dr Pascal Lafôret and Pr Jean-Marie Saudubray, as well as the research laboratory from Pr Alexis Brice for great brainstorming. The authors also wishes to thank patients for their confidence.

\section{Disclosure of interest}

The author declares that she has no conflicts of interest concerning this article. 


\section{Figure legends}

Figure 1. MRI patterns evocative of mitochondrial energy disorders: (a, b) bilateral hyperintensities of the putamen, caudate and thalami called Leigh syndrome from a patient with a mitochondrial DNA mutation; and (c,d) asymmetric cortico-subcortical lesion in the occipital lobe called pseudo-stroke from a patient with POLG mutations.

Figure 2. MRI patterns of neurodegeneration with brain iron accumulation: $(a, b)$ bilateral T2 hypointensity of the globus pallidus (a) and substantia nigra (b) from a patient with PLA2G6 mutations; (c,d) hypointensity of the substantia nigra on axial T2* scan (c) surrounded by a T1 hyperintense halo (d) from a patient with WDR45 mutations. 


\section{References}

[1] Lamari F, Mochel F, Saudubray JM. An overview of inborn errors of complex lipid biosynthesis and remodelling. J Inherit Metab Dis. 2014 Sep 20.

[2] Yu-Wai-Man P, Griffiths PG, Gorman GS, Lourenco CM, Wright AF, Auer-Grumbach M, et al. Multi-system neurological disease is common in patients with OPA1 mutations. Brain. 2010 Mar;133(Pt 3):771-86.

[3] Cadieux-Dion M, Turcotte-Gauthier M, Noreau A, Martin C, Meloche C, Gravel M et al. Expanding the clinical phenotype associated with ELOVL4 mutation: study of a large FrenchCanadian family with autosomal dominant spinocerebellar ataxia and erythrokeratodermia. JAMA Neurol. 2014 Apr;71(4):470-5.

[4] Sedel F, Lyon-Caen O, Saudubray JM. Therapy insight: inborn errors of metabolism in adult neurology--a clinical approach focused on treatable diseases. Nat Clin Pract Neurol. 2007 May;3(5):279-90.

[5] Krishna SH, McKinney AM, Lucato LT. Congenital genetic inborn errors of metabolism presenting as an adult or persisting into adulthood: neuroimaging in the more common or recognizable disorders. Semin Ultrasound CT MR. 2014 Apr;35(2):160-91.

[6] Kruer MC, Boddaert N, Schneider SA, Houlden H, Bhatia KP, Gregory A, et al. Neuroimaging features of neurodegeneration with brain iron accumulation. AJNR Am J Neuroradiol. 2012 Mar;33(3):407-14.

[7] Colombelli C, Aoun M, Tiranti V. Defective lipid metabolism in neurodegeneration with brain iron accumulation (NBIA) syndromes: not only a matter of iron. J Inherit Metab Dis. 2015 Jan;38(1):123-36.

[8] Di Donato I, Banchi S, Federico A, Dotti MT. Adult-onset genetic leukoencephalopathies. Focus on the more recently defined forms. Curr Mol Med. 2014 Oct 10.

[9] Sedel F, Barnerias C, Dubourg O, Desguerres I, Lyon-Caen O, Saudubray JM. Peripheral neuropathy and inborn errors of metabolism in adults. J Inherit Metab Dis. 2007 Oct;30(5):642-53.

[10] Zhang H, Wang Y, Lin N, Yang R, Qiu W, Han L et al. Diagnosis of Niemann-Pick disease type $C$ with 7-ketocholesterol screening followed by NPC1/NPC2 gene mutation confirmation in Chinese patients. Orphanet J Rare Dis. 2014 Jun 10;9:82. 
[11] Debs R, Depienne C, Rastetter A, Bellanger A, Degos B, Galanaud D et al. Biotinresponsive basal ganglia disease in ethnic Europeans with novel SLC19A3 mutations. Arch Neurol. 2010 Jan;67(1):126-30.

[12] Clot F, Grabli D, Cazeneuve C, Roze E, Castelnau P, Chabrol B et al. Exhaustive analysis of BH4 and dopamine biosynthesis genes in patients with Dopa-responsive dystonia. Brain. 2009 Jul;132(Pt 7):1753-63.

[13] Gras D, Roze E, Caillet S, Méneret A, Doummar D, Billette de Villemeur T et al. GLUT1 deficiency syndrome: an update. Rev Neurol (Paris). 2014 Feb;170(2):91-9.

[14] Cartier N, Hacein-Bey-Abina S, Bartholomae CC, Veres G, Schmidt M, Kutschera I, et al. Hematopoietic stem cell gene therapy with a lentiviral vector in $X$-linked adrenoleukodystrophy. Science. 2009 Nov 6;326(5954):818-23.

[15] Yahalom G, Tsabari R, Molshatzki N, Ephraty L, Cohen H, Hassin-Baer S. Neurological outcome in cerebrotendinous xanthomatosis treated with chenodeoxycholic acid: early versus late diagnosis. Clin Neuropharmacol. 2013 May-Jun;36(3):78-83.

[16] Patterson MC, Vecchio D, Prady H, Abel L, Wraith JE. Lancet Neurol. 2007 Sep;6(9):76572. Miglustat for treatment of Niemann-Pick C disease: a randomised controlled study.

[17] Goizet C, Boukhris A, Durr A, Beetz C, Truchetto J, Tesson C et al. CYP7B1 mutations in pure and complex forms of hereditary spastic paraplegia type 5. Brain. 2009 Jun;132(Pt 6):1589-600.

[18] Garcia-Cazorla A, Mochel F, Lamari F, Saudubray JM. The clinical spectrum of inherited diseases involved in the synthesis and remodeling of complex lipids. A tentative overview. J Inherit Metab Dis. 2014 Nov 21.

[19] Sidransky E, Nalls MA, Aasly JO, Aharon-Peretz J, Annesi G, Barbosa ER et al. Multicenter analysis of glucocerebrosidase mutations in Parkinson's disease. N Engl J Med. 2009 Oct 22;361(17):1651-61.

[20] Mazzulli JR, Xu YH, Sun Y, Knight AL, McLean PJ, Caldwell GA et al. Gaucher disease glucocerebrosidase and $\alpha$-synuclein form a bidirectional pathogenic loop in synucleinopathies. Cell. 2011 Jul 8;146(1):37-52.

[21] Dainese L, Monin ML, Demeret S, Brochier G, Froissart R, Spraul A, et al. Abnormal glycogen in astrocytes is sufficient to cause adult polyglucosan body disease. Gene. 2013 Feb $25 ; 515(2): 376-9$. 


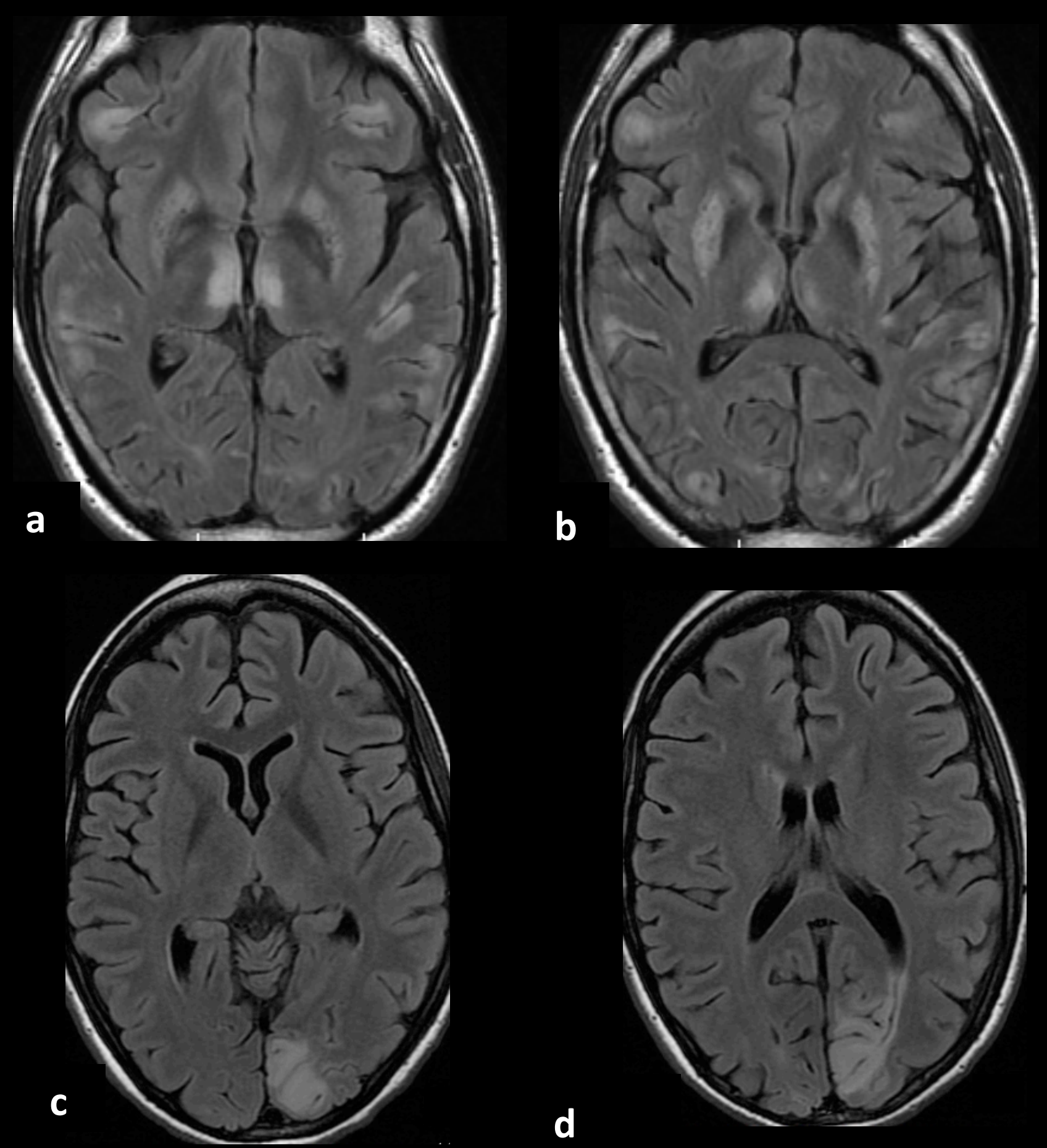



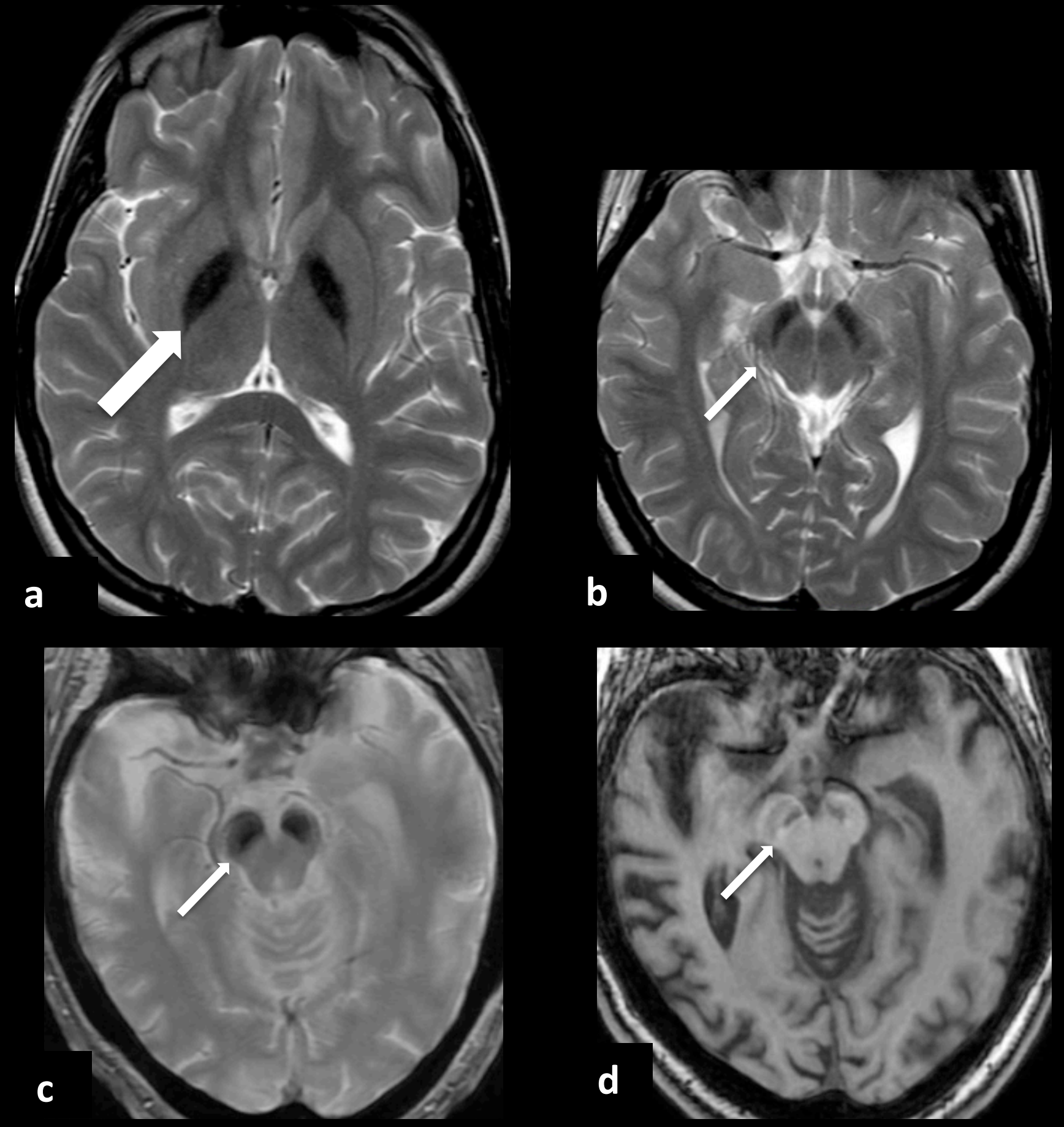